\title{
S-parameter and vector decay constant in QCD with eight fundamental fermions
}

\section{Yasumichi Aoki $^{* a}$, Tatsumi Aoyama ${ }^{a}$, Ed Bennett ${ }^{b, a}$, Masafumi Kurachic ${ }^{c}$, Toshihide Maskawa $^{a}$, Kohtaroh Miura $^{d, a}$, Kei-ichi Nagai $^{a}$, Hiroshi Ohki ${ }^{e}$, Enrico Rinaldi ${ }^{f}$, Akihiro Shibata $^{g}$, Koichi Yamawaki $^{a}$ and Takeshi Yamazaki $^{h}$ (LatKMI Collaboration)}

${ }^{a}$ Kobayashi-Maskawa Institute for the Origin of Particles and the Universe (KMI), Nagoya University, Nagoya 464-8602, Japan

${ }^{b}$ Department of Physics, Swansea University, Singleton Park, Swansea SA2 8PP, UK

${ }^{c}$ Institute of Particle and Nuclear studies, High Energy Accelerator Research Organization (KEK), Tsukuba 305-0801, Japan

${ }^{d}$ Centre de Physique Theorique(CPT), Aix-Marseille University, Campus de Luminy, Case 907, 163 Avenue de Luminy, 13288 Marseille cedex 9, France

${ }^{e}$ RIKEN BNL Research Center, Brookhaven National Laboratory, Upton, NY 11973, USA

${ }^{f}$ Nuclear and Chemical Sciences Division, Lawrence Livermore National Laboratory, Livermore, CA 94550, USA

${ }^{g}$ Computing Research Center, High Energy Accelerator Research Organization (KEK), Tsukuba 305-0801, Japan

${ }^{h}$ Graduate School of Pure and Applied Sciences, University of Tsukuba, Tsukuba, Ibaraki 305-8571, Japan

\begin{abstract}
SU(3) gauge theory with eight massless fundamental fermions seems to be near the conformal boundary, and is a candidate theory of walking technicolor. Along the series of study by LatKMI collaboration using HISQ fermions, $S$-parameter and vector decay constant, which provide important constraints in the model of electroweak symmetry breaking, are calculated for this theory. Use of various volumes allows a systematic investigation of finite volume effects. A strong sensitivity of the $S$-parameter to the volume is found.
\end{abstract}

The 33rd International Symposium on Lattice Field Theory

14 - 18 July 2015

Kobe International Conference Center, Kobe, Japan

\footnotetext{
*Speaker.
} 


\section{Introduction}

Many-flavor QCD provides a possibility for the strong dynamics realization of the Higgs sector in the Standard Model. Among the tested models, SU(3) gauge theory with eight mass-less fundamental fermions has drawn much attention recently ${ }^{1}$, especially as it could be a successful candidate of walking technicolor theory with a flavor-singlet scalar possibly light enough to realize $125 \mathrm{GeV}$ Higgs [2]. In addition to the tests of walking dynamics and light Higgs realization, the Peskin-Takeuchi $S$ parameter [3] provides an important constraint. As increasing number of fermions would naturally enhance $S$, there is a concern that many-flavor models will critically face the limit set by electroweak precision measurements.

The $S$ parameter in the many flavor theories has been studied first by using Bethe-Salpeter equation [4, 5]. Lattice computation of the $S$ parameter is first performed using the overlap fermions [6] and domain-wall fermions [7] on the conventional QCD configurations, mainly aiming to test a methodology. Chiral symmetry at finite lattice spacing is essential for the computation, which is the reason why the chiral fermion formulation is used in these studies.

In the context of strong dynamics theory for the electroweak symmetry breaking, LSD collaboration is the first to systematically study the $S$ parameter on the dependence to the number of flavors $N_{f}$ adopting the domain-wall-fermion formulation in lattice gauge computation. The results are reported for $\mathrm{SU}(3)$ gauge theory with $N_{f}=2,6$ [8] and 8 fundamental fermions [9]. LSD results indicate a decreasing trend of $S$ towards lighter fermion mass $m_{f}$ for $N_{f}=6$ and 8 , which would ease the model building with these theories. The trend is also backed up by the tendency of the observed spectrum towards parity doubling. One caveat, if any, that these studies have is that only one spatial volume is used thus the finite volume effects might have been overlooked. This is particularly important in this context as the finite volume effect tends to reduce the degree of chiral symmetry breaking, which could drive the system towards parity doubling, hence reduce $S$.

We have been studying $N_{f}=8 \mathrm{SU}(3)$ gauge theory with hadron spectrum as a main probe, where the indication of the theory being close to the conformal phase boundary and a parametrically light flavor singlet scalar are found $[10,2,11]$. Therefore, we regard $N_{f}=8 \mathrm{SU}(3)$ gauge theory as a candidate of walking technicolor theory, which has a potential to coop with the experiments. We use the highly-improved staggered quarks (HISQ) throughout our many flavor studies for $N_{f}=4$, $8,12,16$. To fully utilize the gauge configuration ensembles so far accumulated to a good statistics, staggered-fermion calculation for the $S$ parameter is required. We developed the formulation and reported initial results in the past lattice conferences ${ }^{2}$. Here we report on our computation of the $S$ parameter for $N_{f}=8$ and provide an update.

The calculation method, which makes use of the exact chiral symmetry on staggered fermions with multiple staggered field, is briefly described and the preliminary results will be reported in the next section. As a byproduct of the measurements of $S$ parameter, the vector meson decay constant is also measured and reported.

\footnotetext{
${ }^{1}$ see for example reviews by Hasenfratz (these proceedings) and Kuti [1].

${ }^{2}$ Lattice 2013 talk by Y. Aoki and Lattice 2014 talk by H. Ohki.
} 


\section{Vacuum Polarization Functions and Spectrum}

Peskin-Takeuchi $S$ parameter is calculated from the slope of the transverse $V-A$ vacuum polarization function $\Pi\left(q^{2}\right)$ with respect to the infinitesimal squared momentum transfer $q^{2}$,

$$
\begin{aligned}
S & =4 \pi \Pi^{\prime}\left(q^{2}=0\right), \\
\Pi_{\mu v}(q) & =\delta_{\mu v} \Pi\left(q^{2}\right)-\frac{q_{\mu} q_{v}}{q^{2}} \tilde{\Pi}\left(q^{2}\right), \\
\Pi_{\mu v}(q) & \equiv \int d x^{4} e^{i q x}\left\{\left\langle V_{\mu}^{(1,2)}(x) V_{v}^{(2,1)}(0)\right\rangle-\left\langle A_{\mu}^{(1,2)}(x) A_{v}^{(2,1)}(0)\right\rangle\right\},
\end{aligned}
$$

where $V_{\mu}^{(i, j)}$ and $A_{\mu}^{(i, j)}$ are the flavor non-singlet vector and axial vector current respectively, made of anti-quark field with $i$-flavor and quark field with $j$-flavor. This defines the contribution of $S$ parameter from one electro-weak doublet.

On the lattice a naive definition of $S$ parameter may easily suffer from ultra-violet powerdivergent contribution due to a lack of continuum symmetry[6]. An exact symmetry which transforms $V_{\mu}$ into $A_{\mu}$ and vice versa can be used to cancel the UV divergence in the difference. In the literature overlap fermions or domain-wall fermions are used for the computation of $S$ parameter $[6,7,8,9]$ due to this reason. However such calculations are computationally expensive.

As we use staggered fermions, namely HISQ, it is useful to have a formulation to calculate $S$ parameter within HISQ. Staggered fermions have a exact flavor and chiral symmetry which is part of the full flavor and chiral symmetry in the continuum. This flavor symmetry is of the baryon number. The associated current is the flavor singlet vector current in four-flavor (one-species) theory, thus is not suitable to compute the $S$ parameter. Fortunately our primary interest is for the eight flavors, where the exact symmetry enhances to contain $S U(2)_{L} \times S U(2)_{R}$. Therefore the flavor non-singlet non-renormalized vector and axialvector currents can be constructed as the Noether currents of the symmetry.

The conserved currents of HISQ involve length-one and three point-split bilinears. All have the same spin-taste structures

$$
\left(\gamma_{\mu} \otimes 1\right) \tau^{a},\left(\gamma_{\mu} \gamma_{5} \otimes \xi_{5}\right) \tau^{a}
$$

for vector and axial vector respectively. $\tau^{a}$, being $S U(2)$ generators for the eight-flavor theory, bind two different staggered fields. The length-three operators are from the Naik term in the action. Apart from the spin-taste structure, the vector and the axial vector currents are exactly same. The exact chiral symmetry with the generator $\left(\gamma_{5} \otimes \xi_{5}\right) \tau^{a}$ transforms one into another. It is this transformation property that ensures the cancellation of the UV power divergence when the $V-A$ difference of the vacuum polarization function is composed. Therefore, the use of the conserved current is not necessary in general. For example, the operator with just the length-one part from the conserved current will do the job, though renormalization of the operator is then necessary.

In practice we chose to use the one-link point-split currents at the source position of the twopoint function and the full conserved currents at the sink. This makes both the numerical effort and coding complexity minimal while keeping useful Ward-Takahashi identities intact. The necessary renormalization factor for the one-link operator is calculated by taking the ratio:

$$
Z_{A}(t)=\frac{\left\langle\mathscr{A}_{4}(t) P(0)\right\rangle}{\left\langle A_{4}(t) P(0)\right\rangle}
$$


where $\mathscr{A}_{4}(t)$ and $A_{4}(t)$ are the temporal component of the conserved and one-link axial vector current respectively with zero-momentum projection at time $t . P(0)$ is the pseudoscalar operator with the structure $\left(\gamma_{5} \otimes \xi_{5}\right)$ at the origin. The axial current renormalization $Z_{A}$ for $\mathscr{A}_{\mu}=Z_{A} A_{\mu}$ is determined at plateau at large $t$. The left panel of Figure 1 shows the ratio $Z_{A}(t)$ for the so-
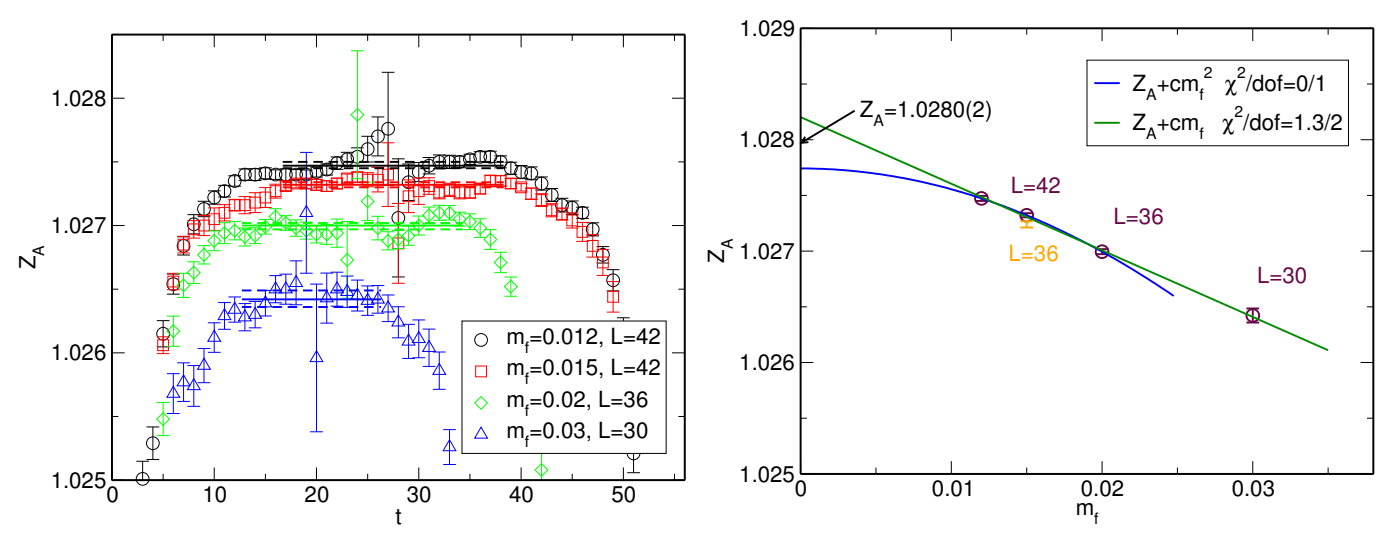

Figure 1: Ratio and plateau fitting for $Z_{A}$ (left) and chiral extrapolation with linear or quadratic in $m_{f}$ (right).

far smallest masses. The right panel shows the extrapolation to the chiral limit using these four points, where we use the largest volume for each mass $L=30\left(m_{f}=0.03\right), 36\left(m_{f}=0.02\right)$ and $42\left(m_{f}=0.015\right.$ and 0.0125$)$ for $L^{3} \times T(T=4 / 3 * L)$ lattice. Both linear extrapolation with all four points and quadratic with lightest three are good. We adopt the average for our $Z_{A}$ and the systematic error of the extrapolation is estimated from the difference from the average to linear or quadratic, which is small $(0.02 \%)$. The statistical error is less than $0.005 \%$, thus negligible.

For the $S$-parameter, vacuum polarization from the vector current two-point function is needed, too. The one-link vector current is renormalized with $Z_{A}$ so far obtained by taking advantage of the exact chiral symmetry, which leads to $Z_{V}=Z_{A}$.

The results of $V-A$ transverse vacuum polarization function $\Pi\left(q^{2}\right)$ are shown in Fig. 2. These results are from the bare two point functions. The one-link operator at the source position needs to be renormalized, which will be taken care of by multiplying one factor of $Z_{A}$ to the results. The Solid lines show fits with the Padé approximation $f\left(q^{2}\right)=\left(b_{0}+b_{1} q^{2}\right) /\left(1+c_{1} q^{2}+c_{2} q^{4}\right)$ [8].

The strong-dynamics contribution to the $S$ parameter per electroweak doublet, Eq. (2.1) is calculated through the Padé fit and shown in Fig. 3 as a function of quark mass $m_{f}$ (left panel). Multiplication of $Z_{A}$ has been done to get renormalized $S$ parameter. Following the same color toward smaller $m_{f}, S$ bends down. This movement is of a finite volume effect as changing to larger volume will move the point back upward. The size of the effect is quite large. For example, the change of $S$ at $m_{f}=0.015$ from $L=36$ to 42 is $8 \%$, while the pion mass shift at the same point is only $0.04 \%$ and statistically consistent with zero. Note that for the test against experiment, subtraction of the standard Higgs sector from this result is needed [3, 12]. The finite volume effect discussed here, however, is independent from that.

The right panel drawn against $m_{\pi} L$, a dimension-less product of techni-pion mass $m_{\pi}$ and the linear spatial size $L$, shows a same structure for different $L$. The left-most points of $L=18$ and 36 suffer from the finite volume effect, which is apparent from the left panel. The bending-down 


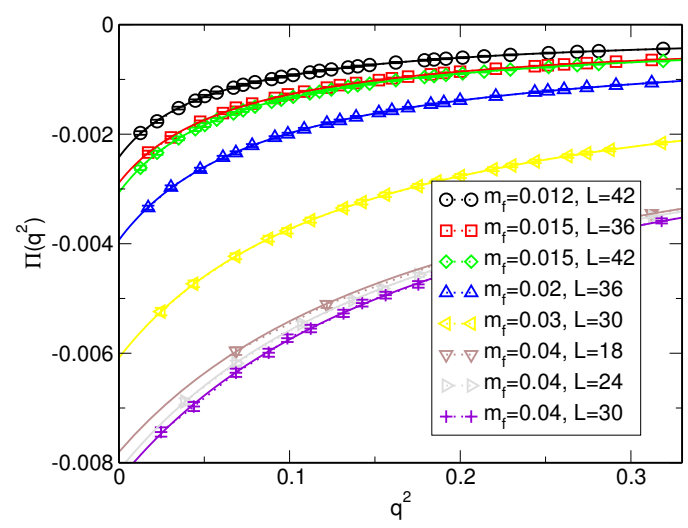

Figure 2: Bare $V-A$ transverse vacuum pluralization function $\Pi\left(q^{2}\right)$. Solid lines show fits with the Padé approximation $f\left(q^{2}\right)=\left(b_{0}+b_{1} q^{2}\right) /\left(1+c_{1} q^{2}+c_{2} q^{4}\right)$.
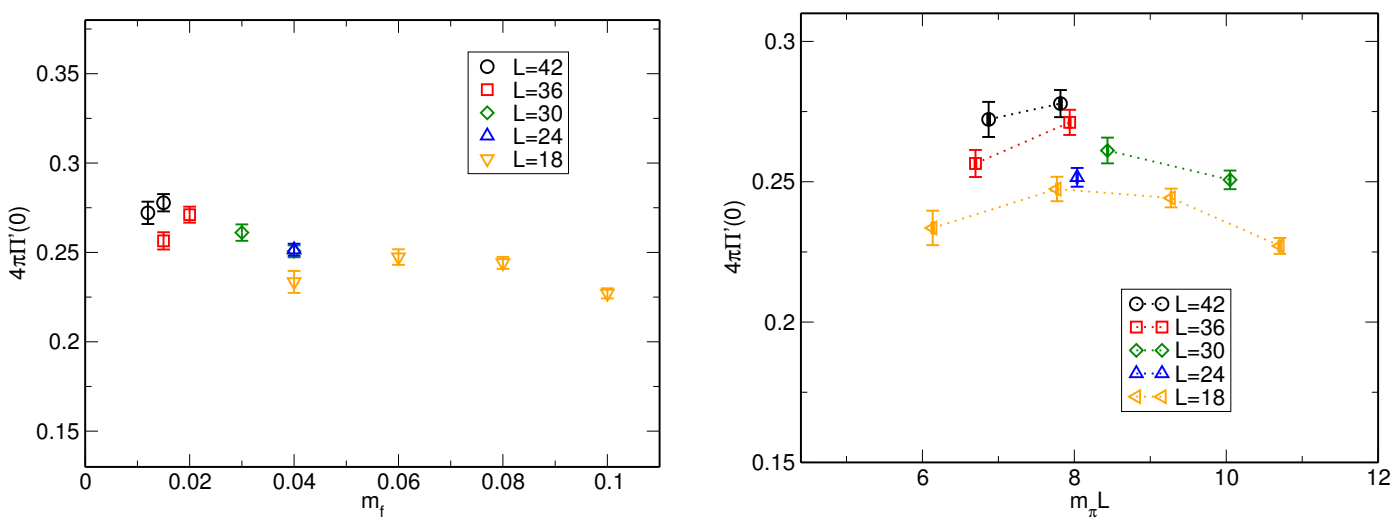

Figure 3: Strong-dynamics contribution to the $S$ parameter per electroweak doublet as a function of quark mass $m_{f}$ (left) or of a dimension-less product of techni-pion mass $m_{\pi}$ and the linear spatial size $L$ (right).

seems to occur around $m_{\pi} L \simeq 7$. As the smallest mass point $m_{f}=0.012$ with $L=42$ is in the range of $m_{\pi} L<7$, the finite volume effect is suspected.

The left panel of Fig. 4 shows the vector and axialvector meson masses measured with the PV channel $\left(\gamma_{k} \gamma_{4} \otimes \xi_{k} \xi_{4}\right)$. Although this channel is different from the one that is used to calculate $S$, we use this as the signal is much better than that due to the locality of the operator in the staggered hypercube. We expect that good taste symmetry, which has been confirmed in the pion sector, also holds for the these sectors.

A remarkable finite volume dependence is observed for the vector meson mass, which drives the meson heavier as the volume is reduced. Due to the larger statistical noise the situation is not very clear for the axialvector. However for $m_{f}=0.02$ and 0.03 , the effect appear opposite to the vector meson. The movement to the small volume is towards the parity doubling, thus seems consistent with the volume effect on $S$ through the dispersive analysis using spectral decomposition [3]. We have to note, however, the correspondence between the parity doubling and the decrease of $S$ is not entirely clear with the current result. For example, as already noted $S$ decreased $8 \%$ from 

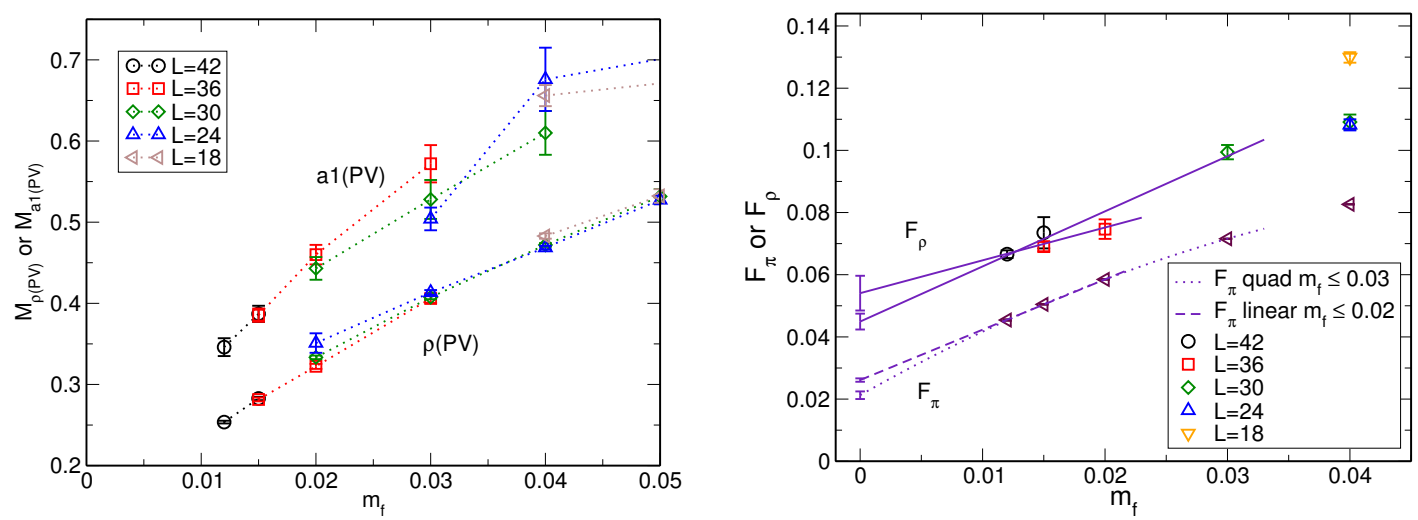

Figure 4: Vector $(\rho)$ and axialvector $\left(a_{1}\right)$ meson masses measured with $P V$ channel as a function of quark mass (left). Vector meson decay constant through the smeared one-link current is plotted (colored symbols) in comparison with the pion decay constant (black symbols) (right). Linear extrapolation for the chiral limit $F_{\rho}$ is performed with two different mass ranges.

$L=42$ to 36 at $m_{f}=0.015$, while the meson masses at the same parameter are stable.

In the spectral decomposition of the $S$ parameter [3] not only the mass but also the decay constant enters. The right panel of Fig. 4 shows the vector meson decay constant computed through the symmetric two-point function with the one-link operator in both source and sink position. There, significant volume dependence is not observed except for the smallest volume, $L=18$.

One also needs to study the axialvector decay constant, which is not shown in this report and remains to be a future task. With our data the vector and axialvector decay constant can in principle be determined. However the axial vector channel is an excited state in the two point correlation function, therefore quite noisy. This is, unfortunately, a drawback of the use of staggered fermions.

Let us get back to the vector decay constant and try to infer the value in the chiral limit. A rough analysis leads to the chiral limit value of the decay constant which appear approximately twice as the pion decay constant $f_{\rho} \simeq 2 f_{\pi}$. This is consistent with the results obtained by LSD collaboration [9] with domain-wall fermions.

\section{Summary and Outlook}

In this report we briefly described how the Peskin-Takeuchi $S$ parameter is computed using Highly Improved Staggered Quarks (HISQ) and preliminary results obtained by applying the method to the eight-flavor SU(3) gauge theory were shown. A remarkable strong sensitivity of the $S$ parameter to the system volume was found, which indicates a careful volume study is indispensable to obtain the correct chiral behavior of the $S$ parameter. The spectrum closely related with $S$ parameter includes vector and axialvector meson masses and decay constants. Noise reduction, where a brute-force method might suffice, especially on the axialvector sector will be useful for an in-depth understanding of the finite volume effect on $S$. $V-A$ vacuum polarization can be used as a probe of (near) conformality through the mass scaling [13], which will be of interest when the finite volume issues are settled. 
Acknowledgments - Numerical computations have been carried out on $\varphi$ at KMI, CX400 at the Information Technology Center in Nagoya University, and CX400 and HA8000 at the Research Institute for Information Technology in Kyushu University. This work is supported by the JSPS Grant-in-Aid for Scientific Research (S) No.22224003, (C) No.23540300 (K.Y.), for Young Scientists (B) No.25800139 (H.O.) and No.25800138 (T.Y.), and also by the MEXT Grants-in-Aid for Scientific Research on Innovative Areas No.23105708 (T.Y.) and No.25105011 (M.K.). This work is supported by the JLDG constructed over the SINET of NII. The work of H.O. is supported by the RIKEN Special Postdoctoral Researcher program. E.R. acknowledges the support of the U.S. Department of Energy under Contract DE-AC52-07NA27344 (LLNL).

\section{References}

[1] J. Kuti, The Higgs particle and the lattice, PoS LATTICE2013 (2014) 004.

[2] Y. Aoki, T. Aoyama, M. Kurachi, T. Maskawa, K. Miura, K.-i. Nagai, H. Ohki, E. Rinaldi, A. Shibata, $\mathrm{K}$. Yamawaki, and T. Yamazaki, A light composite scalar in eight-flavor QCD on the lattice, arXiv:1309.0711.

[3] M. E. Peskin and T. Takeuchi, Estimation of oblique electroweak corrections, Phys. Rev. D46 (1992) 381-409.

[4] M. Harada, M. Kurachi, and K. Yamawaki, Enhanced pi+ - piO mass difference and vanishing $S$ in the large N(f) QCD, Prog. Theor. Phys. 115 (2006) 765-795, [hep-ph / 0509193 ].

[5] M. Kurachi and R. Shrock, Behavior of the S Parameter in the Crossover Region Between Walking and QCD-Like Regimes of an SU(N) Gauge Theory, Phys.Rev. D74 (2006) 056003, [hep-ph/0607231].

[6] JLQCD Collaboration, E. Shintani et. al., S-parameter and pseudo-Nambu-Goldstone boson mass from lattice QCD, Phys. Rev. Lett. 101 (2008) 242001, [arXiv: 0806 . 4222].

[7] RBC Collaboration, P. A. Boyle, L. Del Debbio, J. Wennekers, and J. M. Zanotti, The S Parameter in QCD from Domain Wall Fermions, Phys. Rev. D81 (2010) 014504, [arXiv : 0909.4931 ].

[8] LSD Collaboration, T. Appelquist et. al., Parity Doubling and the S Parameter Below the Conformal Window, Phys. Rev. Lett. 106 (2011) 231601, [arXiv:1009.5967].

[9] LSD Collaboration Collaboration, T. Appelquist et. al., Lattice simulations with eight flavors of domain wall fermions in SU(3) gauge theory, Phys.Rev. D90 (2014), no. 11 114502, [arXiv:1405.4752].

[10] LatKMI Collaboration, Y. Aoki, T. Aoyama, M. Kurachi, T. Maskawa, K.-i. Nagai, H. Ohki, A. Shibata, K. Yamawaki, and T. Yamazaki, Walking signals in $N f=8 Q C D$ on the lattice, Phys.Rev. D87 (2013) 094511, [arXiv:1302.6859].

[11] Y. Aoki, T. Aoyama, M. Kurachi, T. Maskawa, K. Miura, K.-i. Nagai, H. Ohki, E. Rinaldi, A. Shibata, K. Yamawaki, and T. Yamazaki, Walking and conformal dynamics in many-flavor QCD, PoS LATTICE 2015 (2016) 213.

[12] LSD Collaboration, D. Schaich, S parameter and parity doubling below the conformal window, PoS LATTICE2011 (2011) 087, [arXiv:1111.4993].

[13] L. Del Debbio and R. Zwicky, Scaling relations for the entire spectrum in mass-deformed conformal gauge theories, Phys.Lett. B700 (2011) 217-220, [arXiv: 1009.2894 ]. 